\title{
Relearning the importance of routines from rugby player Goromaru
}

\author{
Kazuhiro Nishigami ${ }^{1}$
}

(C) The Japan Society of Ultrasonics in Medicine 2015

Keywords Routine - Ultrasound · Goromaru · Point-of-care $\cdot$ Screening

The rousing success of the Japan national team at the Rugby World Cup sent Japan into a frenzy. The routine of Goromaru, in particular, who successfully made kick after kick, attracted tremendous interest among people of all ages. The objective of his routine is to stay calm and always maintain a constant kicking action. In the case of ultrasound, as well, every institution has a routine examination with a minimum number of elements that should be evaluated and specific procedures, even if they may differ slightly from institution to institution.

Routine ultrasound plays a major role in preventing lesions from being overlooked, as well as in standardizing the skill level of operators. However, several requirements need to be satisfied when creating a routine. First, evaluations and procedures that can be performed in a short period of time are needed in order to finish the examination within a limited amount of time. Next, an approach that is not greatly affected by the body type or condition of the subject is necessary. Finally, the examination must include evaluations of pathologic conditions that must not be overlooked. In general, every institution creates its own routine that meets these requirements for each type of ultrasound examination. At my institution, for example, we evaluate the aorta using a 4-step approach during echocardiography to quickly detect unruptured aortic aneurysms, in particular, since a coexisting asymptomatic

Kazuhiro Nishigami

kazuhiro-nishigami@saiseikaikumamoto.jp

1 Department of Critical Care and Cardiology, Saiseikai Kumamoto Hospital, Kumamoto, Japan aortic aneurysm is often present in patients with arteriosclerotic disease such as coronary artery disease [1].

Point-of-care ultrasound can be thought of as a routine ultrasound examination performed outside the examination room by someone other than an ultrasound technician. This routine is very important in emergency rooms and intensive care units, in particular. In the United States and Europe, many types of point-of-care ultrasound examination have been advocated and are being used in clinical settings, such as focused assessment with sonography for trauma (FAST) [2], rapid ultrasound in shock (RUSH) [3], and focus assessed transthoracic echocardiography (FATE) [4]. I suggested EASY screening [5] as a form of point-of-care cardiovascular ultrasound for chest pain. Here, I will focus on aortic dissection, pulmonary embolism, and acute coronary syndrome. William Osler's maxim "Life's tragedy is usually arterial" is well known, but "Life's tragedy is usually vascular" may be more appropriate as venous thromboembolism, including pulmonary embolism of not only the arterial system but also the venous system, has become a problem today. In addition to these three disorders, tension pneumothorax is a serious and often overlooked disorder with killer chest pain. It is now possible to diagnosis pneumothorax based on the disappearance of lung sliding or the seashore sign on lung ultrasound [6]. If diagnosis of pneumothorax by lung ultrasound is added to EASY screening to form EASY plus screening, the four killer chest pains can be covered.

In the past, nobody paid much attention to routine ultrasound examinations, but it is my guess that not a small number of members of JSUM thought of the routine of ultrasound examinations as they watched Goromaru's routine attract so much attention across Japan. Like rugby, the routine is an important element of ultrasound examinations, as well. In fact, it is not a stretch to say that the 
level of the routine reflects the quality of the institution as a whole. Although the routine may differ from institution to institution given institutional idiosyncrasies, I feel that the time has come to discuss an average routine with respect to the standard ultrasonography recommended by JSUM.

The moment the ball sails beautifully through the uprights after Goromaru has performed his routine is truly a sight to behold. If we are able to contribute to the wellbeing of a patient by discovering an unexpected condition thanks to a routine examination, I think that is the moment that we feel the true pleasure of working as a healthcare professional.

\section{References}

1. Nishigami K. Simultaneous examination of the aorta in echocardiography of patients with coronary artery disease. J Echocardiogr. 2010;8:150-1.
2. American College of Emergency Physicians. Emergency ultrasound imaging criteria compendium. Ann Emerg Med. 2006;48:487-510.

3. Rose JS, Bair AE, Mandavia D, et al. The UHP ultrasound protocol: a novel ultrasound approach to the empiric evaluation of the undifferentiated hypotensive patient. Am J Emerg Med. 2001;19:299-302.

4. Jensen MB, Sloth E, Larsen KM, et al. Transthoracic echocardiography for cardiopulmonary monitoring in intensive care. Eur $\mathbf{J}$ Anaesthsiol. 2004;21:700-7.

5. Nishigami K. Point-of-care echocardiography for aortic dissection, pulmonary embolism and acute coronary syndrome in patients with killer chest pain: EASY screening focused on the assessment of effusion, aorta, ventricular size and shape and ventricular asynergy. J Echocardiogr. 2015;13:141-4.

6. Lichtenstein DA, Menu Y. A bedside ultrasound sign ruling out pneumothorax in the critically ill. Lung sliding. Chest. 1995;108: 1345-8. 